\title{
A personal context-aware multi-device coaching service that supports a healthy lifestyle
}

\author{
Rieks op den Akker and Randy Klaassen \\ Human Media Interaction \\ University of Twente \\ 7500AE Enschede \\ the Netherlands \\ infrieks@ewi.utwente.nl
}

Gijs Geleijnse and Aart van Halteren

Philips Corporate Technologies, Research

5656AE Eindhoven

the Netherlands

www.research.philips.com

aart.van.halteren@philips.com

\author{
Tine Lavrysen \\ Human Interface Group \\ 2800 Mechelen \\ Belgium \\ www.higroup.com \\ tine.lavrysen@higroup.com
}
Henk Schwietert and Marloes van der Hout
Evalan B.V.
1018 AV Amsterdam
the Netherlands
www.evalan.com
henk.schwietert@evalan.com

\begin{abstract}
This paper describes work in progress in the European Artemis project Smarcos. Smarcos focuses on interusability of multi-device embedded and networked services. The work presented here is devoted to the development of context-aware personal coaching service systems that give users personalized feedback to empower them to make healthy lifestyle choices. Our target group consists of diabetes type II patients and the lifestyle intervention concerns situated coaching with respect to physical activity and medicine compliance. We describe the stages in the process, starting with user studies. We describe the ontology that supports the interoperability and the knowledge design to support the coaching strategy, the architecture of the prototype system and the interactions that users have with the system through various (mobile) devices in various contexts. The ultimate aim of the system is to increase the effectiveness of lifestyle interventions by means of interusable coaching interactions. We conclude with an outlook and discussion on the effectiveness of a human-like (autonomous) character as a relational agent representing a virtual coach across devices and situations.
\end{abstract}

e-health, multi-device networked, context-aware, personal, adaptive services, interusability

\section{INTRODUCTION}

This paper describes work in progress carried out in the context of the European Artemis project Smarcos ${ }^{1}$. Smarcos focuses on interusability of multi-device embedded and networked services. Interusability is a central concept in the project, aiming to offer a service in a consistent and recognizable manner at any moment, using any feedback modality on any device. The work presented here is devoted to the development of personal coaching service systems. Such behaviour change support systems provide their users with personalized coaching advice; timely feedback to support them in adhering to a healthy lifestyle. One target group are recently diagnosed diabetes

\footnotetext{
${ }^{1}$ www.smarcos-project.eu
}

II patients. The intervention strategy for these users aims at controlling blood sugar level by means of monitoring user's activities and providing the user context-aware feedback concerning physical activity level and medication compliance, taking into account user's preferences.

We describe the stages in the research process starting with user studies (section 2). Their aim is to investigate users' behaviour and how it diverges from the target behaviour. They result in user and system requirements and a coaching strategy. The Smarcos ontology, aimed at supporting interoperability and the knowledge processing required for implementing the coaching strategies, is described in section 3. In section 4 we describe the architecture of the prototype system and the interactions that users 
have with the system through various (mobile) devices. The prototype system builds on ideas that we find in a number of existing eHealth systems. One example is an Activity Monitor System for COPD patients (Wieringa et al. 2011). They wear a wireless accelerometer that sends the data over Bluetooth to a PDA where further processing and communication to the patient is handled. The system generates feedback messages at fixed time intervals based on the subject's current deviation from a predefined reference line. Another system is Evalan's Real Time Medication Monitoring $\left(\mathrm{RTMM}^{2}\right)$. It has a smart pill dispenser that measures medicine intake and guides users in taking their medicines. When a user forgets to take his medicine, the system sends a notification message by SMS. Feedback by the system improves medication use and therapy adherence of the patients (Vervloet et al. 2011). These two systems are single device coaching systems. The multi-device service system we present here extends the single device feedback systems bringing them on one integrated service platform. It allows multiple contact points with users in various practical situations: at home, at work, on the road. Interventions with several contact points are expected to be more effective in stimulating change in behaviour than those that use a single device (Noar et al. 2007). Social Cognition Models that underly behaviour change strategies -including the Transtheoretical Model of Behaviour Change that we adopted from Prochaska et al. (1994)- emphasize the importance of tailoring health information, such that it is more immediately relevant to an individual. Tailoring refers to "any combination of strategies and information intended to reach one specific person, based on characteristics that are unique to that person, related to the outcome of interest, and derived from an individual assessment" Kreuter et al. (2000) (p. 277). In section 5 we shortly discuss the personalised feedback sent by the central coach.

A key interusability issue is how to present the system to the user in different contexts on different devices. The user interfaces and interaction styles as they are designed for the various devices should be coherent so that the user recognizes that he interacts with one and the same system. In section 6 we consider ins and outs of presenting the coach as an embodied human-like character. Experiments with end users are planned to see how the presentation style of the feedback (by a simple text message or by an embodied human-like agent), is related to interusability of the service and to the effectiveness of the coaching strategy.

${ }^{2}$ www.evalan.com

\section{USE CASES, CONTEXTS, AND USER REQUIREMENTS}

Creating meaningful and effective attentive personal systems implies that these systems provide support taking the context of the user into account, empowering users to take control of their lifestyle. With the ultimate goal to support chronic patients in their daily life, feedback is used to empower users to adopt a certain (more healthy) target behaviour. In order to provide feedback at the right time and using the right modality it is necessary to take into account the context of the user. Therefore, feedback models define the interactions between the attentive personal Smarcos system and the user. To capture the requirements, two context mapping studies have been conducted. Context mapping is a procedure for conducting in-depth research with users. The objective of this procedure is to gain knowledge about the use of products from implied and implicit information that is provided by prospective users during an intensive process (Sleeswijk Visser et al. 2005). Through active participation by users and stakeholders during the design process, it informs and inspires design teams and ensures a good fit between the design and the use of a product. These qualitative studies consisted of a diary booklet with exercises and a semi-structured interview. Over a period of 3 to 5 days, users were probed to describe their daily activities and reflect on their behaviours relevant for the Smarcos system. The study provides insights in the actual behaviour of the users, awareness of the importance of healthful behaviour and their barriers to a desired behaviour. The requirements collected with this approach were further refined by a role playing exercise during a workshop in Amsterdam in which the majority of the Smarcos team members participated. The list of requirements that were identified included the following:

- The system needs to learn the specific Diabetes condition of the user.

- The system needs to know the relevant context information

- The system needs to teach and/ or assist the user about the desired behaviour

- The system needs to be geared towards motivating the user

The feedback models used in the attentive personal Smarcos system are determined by the available sensors, input devices and devices for feedback. A feedback model describes the entire loop from capturing user behaviour, interpretation of inputs and reasoning about desired feedback. For a graphical representation of the basic elements that comprise 
a feedback model, see Figure 1. Figure 2 shows the feedback model for the diabetes use case.
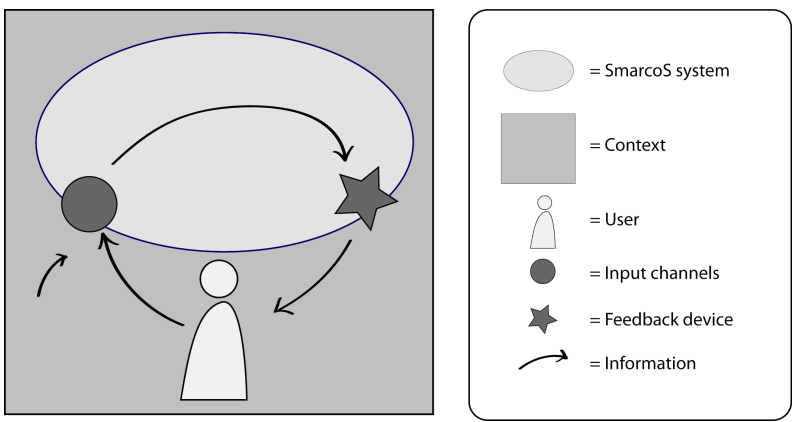

Figure 1: Graphical representation of basic elements for Smarcos feedback models.

Awareness of one's own behaviour and the consequences for health is one of the key processes for health behaviour change (e.g., Prochaska et al. (1994)). To induce improvements in the selected behavioural domains, we focus on creating awareness in a patient's own behaviour and on the personal consequences of the patients' behaviour in terms of blood glucose levels, physical activity and medication intake. To create awareness, simple and meaningful overviews of the patient's behaviours and the outcome variables will be provided to the patient at opportune moments. Moreover, the patient can interact with the system to learn the effects of certain behaviours on the outcome variables in his/her own body. In this way the patient can learn to manage for example blood glucose levels by compensating the effects of certain behaviours through the engagement in other behaviours. To induce behaviour change, we will focus on selfregulation strategies that are offered in a tailored manner. Depending on the level of knowledge of the patient, the stage-of-change the patient is in and the psychological characteristics of the patient, the system can provide informational, motivational or behavioural support to promote behaviour change. We distinguish four different contexts: at home, at work, outside and in the car. In these different Smarcos contexts different devices are available for presenting feedback. Some devices can use different modalities for presenting feedback. Depending on the context and situation of the user the system should select the right device and modalities to present the feedback to the users.

\section{THE SMARCOS ONTOLOGY}

Ontologies are important components of information systems and information processing: they support the interoperability between information systems and avoid isolated applications. They are an important instrument for the modeling of context information in pervasive computing applications. We developed a Smarcos ontology in OWL and RDF using as much as possible existing ontologies, like CONON, an ontology that defines classes to model Person, Location, and Activity.

In order to adapt the personal coaching service to the user's context, information needs to be gathered about that context. A widely accepted definition of context is (citation from Krummenacher and Strang (2007): Context is any information that can be used to characterize the situation of an entity. An entity is a person, place or object that is considered relevant to the interaction between a user and an application, including the user and the application themselves. A system is context-aware if it uses context to provide relevant information and services to the user, where relevancy depends on the users task. The information can be gathered from devices all around the user such as mobile phones, which these days include a large number of sensors such as accelerometers, microphones, light sensors, etc. The context ontology was developed in order to structurally map the hierarchies and relations between elements of the context, the devices in the context and the users themselves. This information will then be used when processing sensor data and inferring what is the most suitable coaching message and feedback modality based on the user's context.

The ontology was constructed by first determining the expected outcome, and then deducing from that which information could be relevant to include in the ontology. The expected outcome was expressed in the form of a list of queries to be answered by the system in order to provide context-sensitive personal coaching. The list included queries about the user's profile (What language does the user speak?, What is the user's home address?, etc), the user's activities (What is the user's physical activity level?, Did the user take his medication at the right time?, etc), the user's current context (What is the user's current physical location?, Which active devices are in the proximity of the user?, etc) and about the device characteristics (Which active devices are able to broadcast textual feedback?, etc). These queries resulted in a taxonomy of relevant elements of the context, devices and user. A simplified version of the context taxonomy can be seen in Figure 3 .

Based on the context, device and user taxonomies, an ontology in OWL was built using Protege. We will extend the ontology with an Ontology for User Preferences regarding activities which can then be used for personalizing feedback provided by the coach in a way similar to (Erriquez and Grasso 2008). 

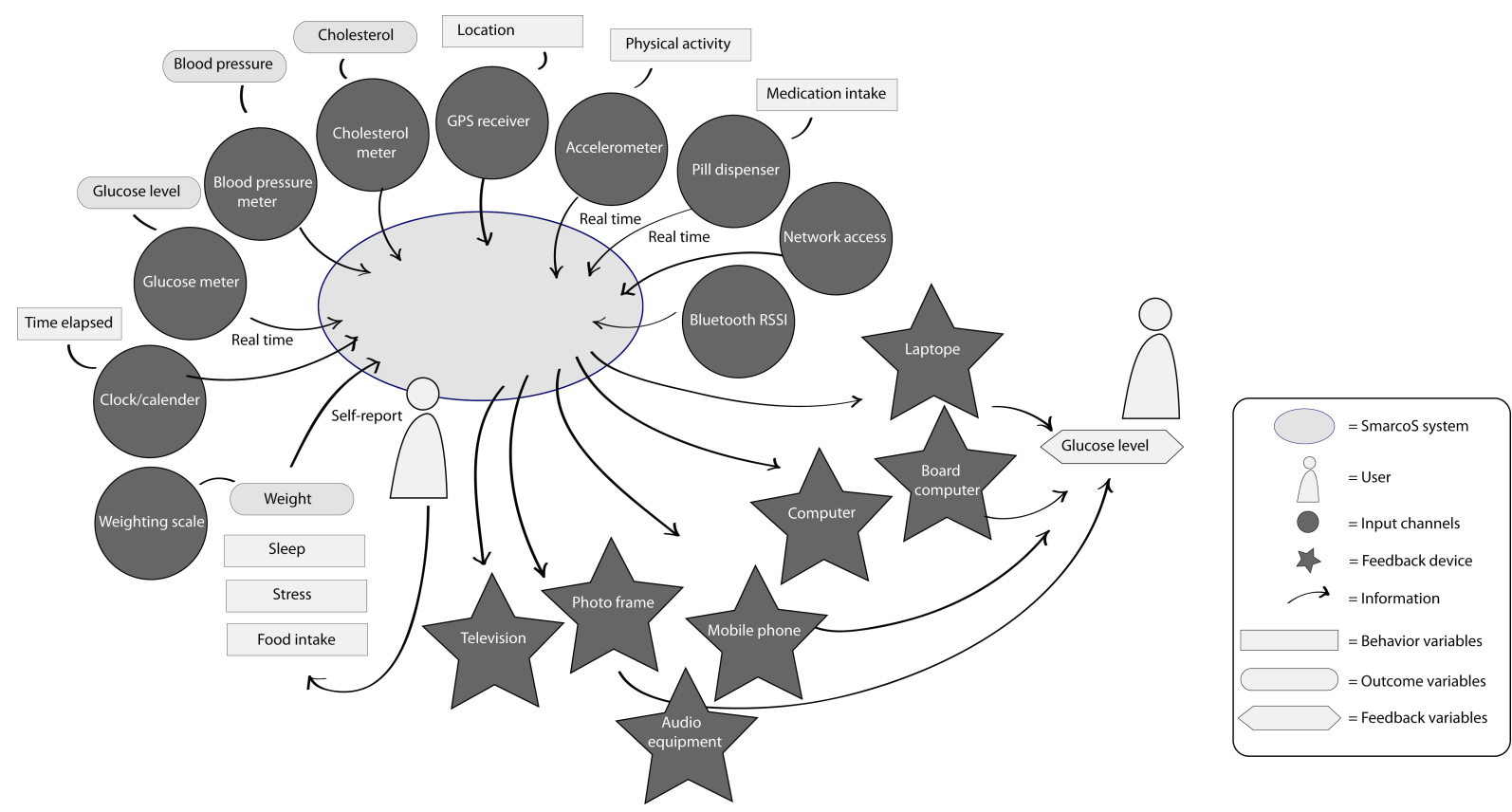

Figure 2: Full model for the diabetes use case.

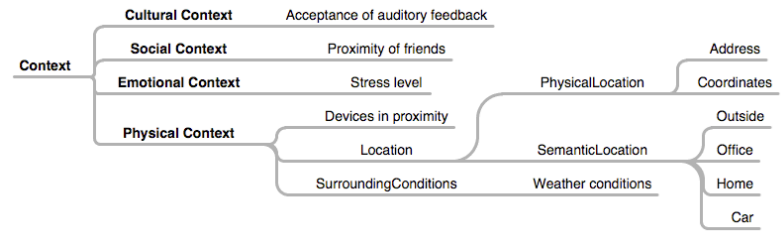

Figure 3: A simplified version of the context part of the Smarcos ontology.

\section{THE REFERENCE ARCHITECTURE}

We follow the shared memory approach adopted by SOFIA, an ongoing European Project and use the SOFIA platform for sharing interoperable information in smart environment applications. This approach decouples information consumers and producers and relevant information is stored on a shared Semantic Information Broker (SIB) accessible by all the relevant actors, the knowledge processors (KP). The SIB provides an interface whose basic components are: join, leave, insert, remove, query and subscribe. The protocol used to communicate with the SIB is the Smart Space Application Protocol, an application layer protocol based on XML. For a KP to interact with the SIB, it must first join the SIB then it can insert or query for information as needed. The interoperability between KPs is provided when each $\mathrm{KP}$ is imbued with the knowledge from the relevant portion of the applications domain ontology. (Vergari et al. 2010)

Figure 4 shows the architecture of the first prototype. On the left side of the diagram two different output devices in different contexts are presented. The right side of the diagram presents the two different input devices of the system. In the middle of the diagram the cloud is represented. All input and output devices are connected to the cloud and have their own KP connected to the SIB. The Semantic Information Broker (SIB) in the cloud stores the Smarcos ontology, and the events. User profile and preference, context information and information sent by the sensors will be updated by the knowledge processors in the SIB. The information in the SIB can be processed with Jena, that provides reasoning engines for inferencing. Based on events and inferences, the coach will present feedback to the user.

\section{COACHING FEEDBACK}

The coaching feedback that the user receives is based on coaching rules executed inside the coaching knowledge processor. This knowledge processor receives updates on the user context from the SIB. The coach considers a number of constraints. These constraints are related to the following aspects:

Timing. When does the coach send a message? What time constraints are there for sending the message? How urgent is the situation? Does it require a message to be sent immediately?

Content. What is the communicative intention of the message? Is this a regular (daily or weekly) report about physical activity, or calory intake? Or is this a warning, an advice, an assessment, or 


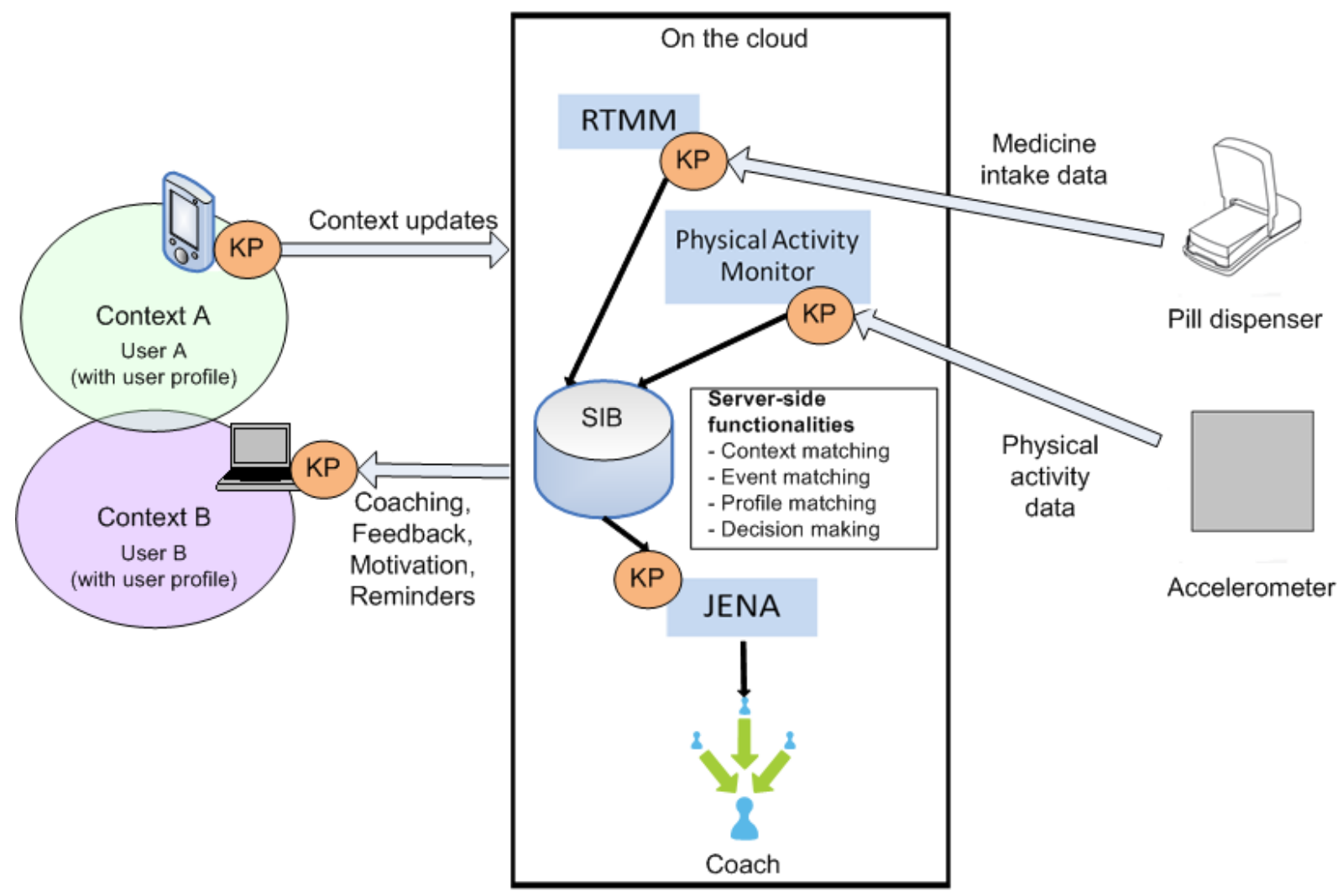

Figure 4: Overall architecture of the coaching system.

maybe a message that asks for specific information from the user? Some existing systems generate feedback messages based on ontologies and knowledge of user preferences and the current situation the user is in. For example does the user like indoor or outdoor activities and what are the weather conditions? (Erriquez and Grasso 2008) Variation of feedback messages is important in order to prevent annoyance. As Bickmore et al. reports: "One surprising finding from the interviews was that, even though the dialogue scripts had been authored to provide significant variability in each days' interaction, most participants found the conversations repetitive at some point during the month. This repetitiveness annoyed subjects, and a few subjects even indicated that it negatively impacted their motivation to exercise..." (Bickmore and Picard 2005).

Modality of Presentation. How is the message presented, on what devices, in what format? Is it spoken by a graphical interface on a mobile phone? Is it a graph showing an overview on a webpage? Is it text that refers to a picture? Is it a sound, a tune that signals the user that he has forgotten to take his pills?

Communication protocol. Is the user required to send a response message in answer to the message?
Should the message be repeated until the user has confirmed receiving the message?

\section{PRESENTING THE COACHING SYSTEM}

Interusability implies that users interacting with the coach through different devices and modalities recognize that they have to do with one and the same service. The coach may be perceived as a relational agent in the sense of Bickmore and Picard (2005): a computational artifacts designed to build long-term socio-emotional relationships with users, including trust, rapport and therapeutic alliance, for the purpose of enhancing adherence to treatment. Presenting the coach by a humanlike character on the interface is expected to support this aspect of interusability. Research on anthropomorphic agent interfaces has produced divergent results (Catrambone et al. 2002) and further experimental research should find out what the effects of personification of the coach are on the way users perceive the system: do users like it? does it imply higher expectations regarding the intelligence of the system than the system can afford?, and on effectiveness of the system: does it have a positive influence on user's trust, on therapy compliance?. 
As soon as we decide to present the Smarcos coach by means of a humanoid the issue pops up what character it (he/she?) should have. What should (s)he look like, sound, etc? Another issue is if we want the user to see the coach as a representation of a human coach or of some organisation ("the Smarcos team") or if he should see it as an autonomous self. For generating multimodal communicative behaviours of the virtual agent, we build on the results of the SAIBA initiative. In particular, we will use the Behaviour Markup Language (BML) for the specification of synchronous multi-modal (speech, gestures, facial expressions) behaviours. We use the Elckerlyc platform (van Welbergen et al. 2010) for the behaviour realization by a character. Rendering fully embodied $3 D$ conversational agents with lifelike expressions is computationally costly. We will find out what is needed for a convincing and persuasive character and what can be realized given the space and realtime constraints imposed by the available devices and network technologies.

\section{CONCLUSION AND FUTURE}

Developing effective multi-device personal coaching systems requires expertise from a number of disciplines and research field including social psychology, eHealth, persuasive technology, user interface design, knowledge engineering, artificial intelligence, natural language dialogue systems, user experience design, and networked services. Due to limited space we could only give a very short impression of the many aspects that the Smarcos team has considered in developing this type of complex persuasive systems. We plan to finish the first prototype and start end user experiments in June. At the conference we expect to present a first prototype of the Smarcos virtual coach as well as some first results.

\section{ACKNOWLEDGMENTS}

Thanks to all Smarcovians who contributed in one way or another to the work reported here. This work was funded by the European Commission, within the framework of the ARTEMIS JU SP8 SMARCOS project 100249 - (www.smarcos-project.eu).

\section{REFERENCES}

Bickmore, T. and Picard, R. (2005). Establishing and maintaining long-term humancomputer relationships. ACM Transactions on Computer-Human Interaction, 12:293-327.

Catrambone, R., Stasko, J., and Xiao, J. (2002). Anthropomorphic agents as a user interface paradigm:
Experimental findings and a framework for research. In Proceedings of the 24th Annual Conference of the Cognitive Science Society, pages 166-171.

Erriquez, E. and Grasso, F. (2008). Generation of personalised advisory messages: An ontology based approach. In Computer-Based Medical Systems, 2008. CBMS '08. 21st IEEE International Symposium on, pages $437-442$.

Kreuter, M. W., Farrell, D., Olevitch, L., and Brennan, L. (2000). Tailoring health messages: Customizing communication with computer technology. Mahwah, $\mathrm{NJ}$ : Erlbaum.

Krummenacher, R. and Strang, T. (2007). Ontologybased context modeling. In In Workshop on ContextAware Proactive Systems.

Noar, S., Benac, C., and Harris, M. (2007). Does tailoring matter? meta-analytic review of tailored print health behavior change interventions. Psychological Bulletin, page 673693.

Prochaska, J. O., Velicer, W. F., Rossi, J. S., Goldstein, M. G., Marcus, B. H., Rakowski, W., Fiore, C., Harlow, L. L., Redding, C. A., Rosenbloom, D., and Rossi, S. R. (1994). Stages of change and decisional balance for twelve problem behaviors. Health Psychology, 13:39-46.

Sleeswijk Visser, F., Stappers, P. J., van der Lugt, R., and Sanders, E. (2005). Contextmapping: experiences from practice. CoDesign, 1(2):119149.

van Welbergen, H., Reidsma, D., Ruttkay, Z. M., and Zwiers, J. (2010). Elckerlyc: A BML realizer for continuous, multimodal interaction with a virtual human. Journal on Multimodal User Interfaces, 3(4):271-284.

Vergari, F., Bartolini, S., Spadini, F., D’Elia, A., Zamagni, G., Roffia, L., and Cinotti, T. S. (2010). A smart space application to dynamically relate medical and environmental information. In Proceedings of the Conference on Design, Automation and Test in Europe, DATE '10, pages 1542-1547, 3001 Leuven, Belgium, Belgium. European Design and Automation Association.

Vervloet, M., van Dijk, L., Santen-Reestman, J., van Vlijmen, B., Bouvy, M., and de Bakker, D. (2011). Improving medication adherence in diabetes type 2 patients through real time medication monitoring. BMC Health Services Research, 11(1):5.

Wieringa, W., op den Akker, H., Jones, V. M., op den Akker, R., and Hermens, H. J. (2011). Ontologybased generation of dynamic feedback on physical activity. In Proceedings AIME'2011. 